\title{
A Morphism Double Category and Monoidal Structure
}

\author{
Saikat Chatterjee, ${ }^{1}$ Amitabha Lahiri, ${ }^{2}$ and Ambar N. Sengupta ${ }^{3}$ \\ ${ }^{1}$ School of Mathematics, Harish Chandra Research Institute, Chhatnag Road, Jhusi, Allahabad, Uttar Pradesh 211 019, India \\ ${ }^{2}$ S. N. Bose National Centre for Basic Sciences, Block JD, Sector III, Salt Lake, Kolkata, West Bengal 700098, India \\ ${ }^{3}$ Department of Mathematics, Louisiana State University, Baton Rouge, LA 70803, USA
}

Correspondence should be addressed to Amitabha Lahiri; amitabhalahiri@gmail.com

Received 15 November 2012; Revised 13 February 2013; Accepted 14 February 2013

Academic Editor: Dae San Kim

Copyright (C) 2013 Saikat Chatterjee et al. This is an open access article distributed under the Creative Commons Attribution License, which permits unrestricted use, distribution, and reproduction in any medium, provided the original work is properly cited.

We provide a recipe for "fattening" a category that leads to the construction of a double category. Motivated by an example where the underlying category has vector spaces as objects, we show how a monoidal category leads to a law of composition, satisfying certain coherence properties, on the object set of the fattened category.

\section{Introduction and Geometric Background}

The interaction of point particles through a gauge field can be encoded by means of Feynman diagrams, with nodes representing particles and directed edges carrying an element of the gauge group representing parallel transport along that edge. If the point particles are replaced by extended onedimensional string-like objects, then the interaction between such objects can be encoded through diagrams of the form

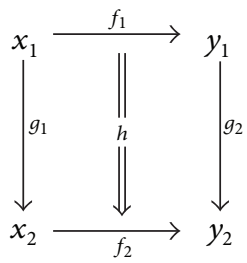

where the labels $f_{i}$ and $g_{i}$ describe classical parallel transport and $h$, which may take values in a different gauge group, describes parallel transport over a space of paths.

We will now give a rapid account of some of the geometric background. We refer to our previous work [1] for further details. This material is not logically necessary for reading the rest of this paper but is presented to indicate the context and motivation for some of the ideas of this paper.

Consider a principal $G$-bundle $\pi: P \rightarrow M$, where $M$ is a smooth finite dimensional manifold and $G$ a Lie group, and a connection $\bar{A}$ on this bundle. In the physical context, $M$ may be spacetime, and $\bar{A}$ describes a gauge field. Now consider the set $\mathscr{P} M$ of piecewise smooth paths on $M$, equipped with a suitable smooth structure. Then, the space $\mathscr{P}_{\bar{A}} P$ of $\bar{A}$ horizontal paths in $P$ forms a principal $G$-bundle over $\mathscr{P} M$. We also use a second gauge group $H$ (that governs parallel transport over path space), which is a Lie group along with a fixed smooth homomorphism $\tau: H \rightarrow G$ and a smooth map

$$
G \times H \longrightarrow H:(g, h) \longmapsto \alpha(g) h
$$

such that each $\alpha(g)$ is an automorphism of $H$, such that

$$
\begin{gathered}
\tau(\alpha(g) h)=g \tau(h) g^{-1}, \\
\alpha(\tau(h)) h^{\prime}=h h^{\prime} h^{-1}
\end{gathered}
$$

for all $g \in G$ and $h, h^{\prime} \in H$. We denote the derivative $\tau^{\prime}(e)$ by $\tau$, viewed as a map $L H \rightarrow L G$, and denote $\alpha^{\prime}(e)$ by $\alpha$, to avoid notational complexity. Given also a second connection form $A$ on $P$ and a smooth $\alpha$-equivariant vertical $L H$-valued 2 -form $B$ on $P$, it is possible to construct a connection form $\omega_{(A, B)}$ on the bundle $\mathscr{P}_{\bar{A}} P$

$$
\omega_{(A, B)}=\mathrm{e} v_{1}^{*} A+\tau(Z),
$$


where $Z$ is the $L H$-valued 1 -form on $\mathscr{P}_{\bar{A}} P$ specified by

$$
Z=\int_{0}^{1} B
$$

which is a Chen integral.

Consider a path of paths in $P$ specified through a smooth map

$$
\widetilde{\Gamma}:[0,1]^{2} \longrightarrow P:(t, s) \longmapsto \widetilde{\Gamma}(t, s)=\widetilde{\Gamma}_{s}(t)=\widetilde{\Gamma}^{t}(s),
$$

where each $\widetilde{\Gamma}_{s}$ is $\bar{A}$-horizontal and the path $s \mapsto \widetilde{\Gamma}(0, s)$ is $A$-horizontal. Let $\Gamma=\pi \circ \widetilde{\Gamma}$. The bi-holonomy $g(t, s) \in G$ is specified as follows: parallel translate $\widetilde{\Gamma}(0,0)$ along $\Gamma_{0} \mid[0, t]$ by $\bar{A}$, then up the path $\Gamma^{t} \mid[0, s]$ by $A$, back along $\Gamma_{s}$-reversed by $\bar{A}$ and then down $\Gamma^{0} \mid[0, s]$ by $A$, then the resulting point is

$$
\widetilde{\Gamma}(0,0) g(t, s)
$$

The following result is proved in [1].

Theorem 1. Suppose that

$$
\widetilde{\Gamma}:[0,1]^{2} \longrightarrow P:(t, s) \longmapsto \widetilde{\Gamma}(t, s)=\widetilde{\Gamma}_{s}(t)=\widetilde{\Gamma}^{t}(s)
$$

is smooth, with each $\widetilde{\Gamma}_{s}$ being $\bar{A}$-horizontal and the path $s \mapsto$ $\widetilde{\Gamma}(0, s)$ being $A$-horizontal. Then, the parallel translate of $\widetilde{\Gamma}_{0}$ by the connection $\omega_{(A, B)}$ along the path $[0, s] \rightarrow \mathscr{P} M: u \mapsto \Gamma_{u}$, where $\Gamma=\pi \circ \widetilde{\Gamma}$, results in

$$
\widetilde{\Gamma}_{s} g(1, s) \tau\left(h_{0}(s)\right),
$$

with $g(1, s)$ being the "bi-holonomy" specified as in (7), and $s \mapsto h_{0}(s) \in H$ solving the differential equation

$$
\begin{aligned}
\frac{d h_{0}(s)}{d s} h_{0}(s)^{-1}= & -\alpha\left(g(1, s)^{-1}\right) \\
& \times \int_{0}^{1} B\left(\partial_{t} \widetilde{\Gamma}(t, s), \partial_{s} \widetilde{\Gamma}(t, s)\right) d t
\end{aligned}
$$

with initial condition $h_{0}(0)$ being the identity in $H$.

Consider the category $\mathbf{C}_{0}$ whose objects are fibers of a given vector bundle $E$ over $M$ and whose arrows are piecewise smooth paths in $M$ (up to "backtrack equivalence"; for more on this notion see [2]) along with parallel transport operators, by a connection $\bar{A}$, along such paths. Note that all arrows are invertible. In Figure $1, E_{p_{1}}$ is the vector space which is the fiber over the corresponding point $p_{1}$. For the path $c_{1}$, there is a parallel transport operator $f_{1}: E_{p_{1}} \rightarrow E_{q_{1}}$. Next, if $c_{2}$ is a path from the base of the fiber $E_{p_{2}}$ to the base of $E_{q_{2}}$, then there is a corresponding parallel transport operator $f_{2}: E_{p_{2}} \rightarrow E_{q_{2}}$.

A "higher" morphism $c_{1} \rightarrow c_{2}$ is obtained from any suitably smooth path of paths, starting with the initial path $c_{1}$ and ending with $c_{2}$ (again backtracks need to be erased). Using the connection $\bar{A}$, this produces parallel transport

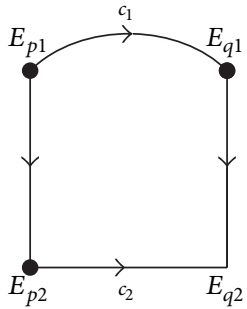

Figure 1: Paths and fibers.

operators and paths $E_{p_{1}} \rightarrow E_{p_{2}}$ and $E_{q_{1}} \rightarrow E_{q_{2}}$. Moreover, another connection $A$ and 2-form $B$, along with a path of paths lead to a linear map $\operatorname{Mor}_{l}\left(E_{p_{1}}, E_{q_{1}}\right) \rightarrow \operatorname{Mor}_{l}\left(E_{p_{2}}, E_{q_{2}}\right)$, where $\operatorname{Mor}_{l}(E, F)$ is the vector space of all linear maps $E \rightarrow$ $F$. We view this, in a "first approximation," as a morphism from the object $\operatorname{Mor}\left(E_{p_{1}}, E_{q_{1}}\right)$ to the object $\operatorname{Mor}\left(E_{p_{2}}, E_{q_{2}}\right)$ (say, mapping all paths from $p_{1}$ to $q_{1}$ to the path $c_{2}$ ). In this paper, we will not develop this framework in full detail (that would build on the theory from our earlier work [1]) but focus on more algebraic aspects and other purely algebraic issues (such as monoidal structures).

Instead of vector bundles, one could also work with the principal bundle $P$ itself, taking as objects of a category $\mathbf{C}_{0}$ all the fibers of the bundle $P$ and as morphisms $f: P_{p} \rightarrow P_{q}$ the $G$-equivariant bijections $P_{p} \rightarrow P_{q}$, where $P_{p}$ and $P_{q}$ are fibers of $P$, over points $p$ and $q$, and paths running from $p$ to $q$.

The interface between gauge theory and category theory, in various forms and cases, has been studied in many works, for instance [1, 3-7]. In the present paper, we extract the abstract essence of some of these structures in a category theory setting, leaving the differential geometry behind as the concrete context. We abstract the process of passing from the point-particle picture to a string-like picture to a functor which generates a category $\mathbb{F}(\mathbf{C})$ from a category C. Proposition 5 describes properties of a natural product operation on the objects of $\mathbb{F}(\mathbf{C})$ when $\mathbf{C}$ is a monoidal category. An excellent review of monoidal categories in relation to topological quantum field theory can be found in [8]. Symmetric monoidal bicategories are discussed in [9] in a context different from ours.

\section{The Fat Category}

Let $\mathbf{C}$ be a category. We define a new category $\mathbb{F}(\mathbf{C})$ as follows. The objects of $\mathbb{F}(\mathbf{C})$ are the morphisms of $\mathbf{C}$. A morphism in $\mathbb{F}(\mathbf{C})$ from the object $x_{1} \stackrel{f_{1}}{\longrightarrow} y_{1}$ to the object $x_{2} \stackrel{f_{2}}{\longrightarrow} y_{2}$ consists of morphisms $x_{1} \stackrel{g_{1}}{\longrightarrow} x_{2}$ and $y_{1} \stackrel{g_{2}}{\longrightarrow} y_{2}$ in $\mathrm{C}$, along with a setmapping

$$
h: \operatorname{Mor}\left(x_{1}, y_{1}\right) \longrightarrow \operatorname{Mor}\left(x_{2}, y_{2}\right)
$$

which maps $f_{1}$ to $f_{2}$ as follows:

$$
h\left(f_{1}\right)=f_{2} .
$$


(In a later section we require that the hom-sets $\operatorname{Mor}(x, y)$ themselves also have algebraic structure that should be preserved by such $h$.) Here is a diagram displaying a morphism $u$ of $\mathbb{F}(\mathbf{C})$ :

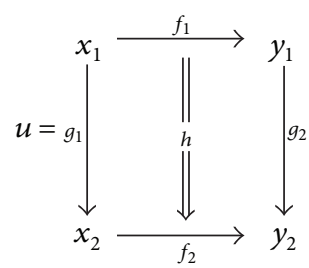

It is clear that this does specify a category, which we call the fat category for C (composition is "vertical," with successive $h$ s composed). Sometimes it will be easier on the eye to write

$$
(x, y, f)
$$

for $x \stackrel{f}{\rightarrow} y$. Thus, diagram (13) can also be displayed as

$$
\begin{gathered}
\left(x_{1}, y_{1}, f_{1}\right) \\
\downarrow_{\left(x_{2}, y_{2}, f_{2}\right)}^{\downarrow}
\end{gathered}
$$

The composition ${ }^{\circ}{ }_{V} u$ of morphisms in $\mathbb{F}(\mathbf{C})$ is defined "vertically" by drawing the diagram of $v$ below that of $u$ and composing vertically downward.

Commutative diagrams in $\mathbf{C}$ lead to morphisms of $\mathbb{F}(\mathbf{C})$ in a natural way and yield a subcategory of $\mathbb{F}(\mathbf{C})$ that is recognizable as the "category of arrows" [10, \$I.4], sometimes denoted as $\operatorname{Arr}(\mathbf{C})$.

Lemma 2. Any commutative diagram

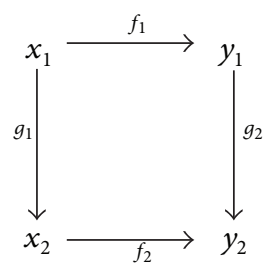

in $\mathbf{C}$, in which $g_{1}$ is an isomorphism, generates a morphism

$$
\left(x_{1}, y_{1}, f_{1}\right) \stackrel{u}{\longrightarrow}\left(x_{2}, y_{2}, f_{2}\right)
$$

in $\mathbb{F}(\mathbf{C})$,

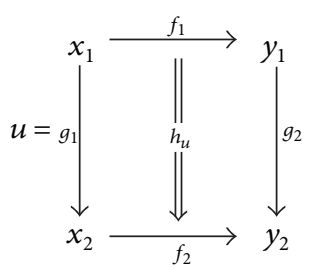

where

$$
h_{u}: \operatorname{Mor}\left(x_{1}, y_{1}\right) \longrightarrow \operatorname{Mor}\left(x_{2}, y_{2}\right): \phi \longmapsto g_{2} \phi g_{1}^{-1}
$$

Moreover, if

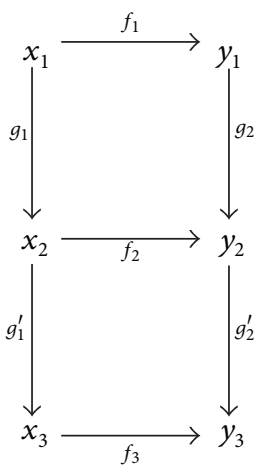

is a commutative diagram in $\mathbf{C}$, where $g_{1}$ and $g_{1}^{\prime}$ are isomorphisms, then the composite of the induced morphisms,

$$
\begin{aligned}
& u:\left(x_{1}, y_{1}, f_{1}\right) \longrightarrow\left(x_{2}, y_{2}, f_{2}\right), \\
& v:\left(x_{2}, y_{2}, f_{2}\right) \longrightarrow\left(x_{3}, y_{3}, f_{3}\right),
\end{aligned}
$$

is the morphism in $\mathbb{F}(\mathbf{C})$ induced by the commutative diagram

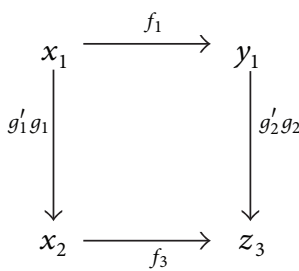

\section{A Double Category of Isomorphisms}

Let $\mathbb{F}(\mathbf{C})_{0}$ be the category whose objects are the invertible arrows of $\mathbf{C}$ and whose arrows are the arrows

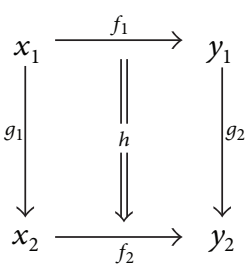

in $\mathbb{F}(\mathbf{C})$ in which the verticals $g_{1}$ and $g_{2}$ are also isomorphisms in $\mathbf{C}$. This is, for all purposes here, as good as assuming that all arrows of $\mathbf{C}$ are invertible, since we will only work with such arrows. In the geometric context, the arrows represent parallel transports and so the invertibility assumption is natural. The mapping $h$ is motivated by the "surface" parallel transport mentioned briefly in (10). 
Let us define horizontal composition of morphisms in $\mathbb{F}(\mathbf{C})_{0}$ as follows:

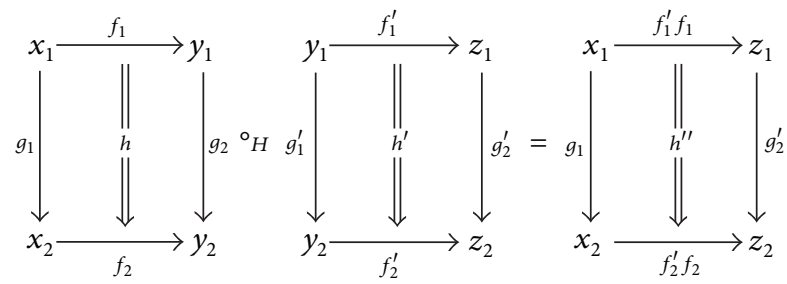

where the composition is defined only when $g_{1}^{\prime}=g_{2}$, and $h^{\prime \prime}$ is given by

$$
h^{\prime \prime}: \operatorname{Mor}\left(x_{1}, z_{1}\right) \longrightarrow \operatorname{Mor}\left(x_{2}, z_{2}\right): f \longmapsto h^{\prime}\left(f f_{1}^{-1}\right) \underbrace{h\left(f_{1}\right)}_{f_{2}} .
$$

Note that $h^{\prime \prime}$ satisfies

$$
h^{\prime \prime}\left(f_{1}^{\prime} f_{1}\right)=h^{\prime}\left(f_{1}^{\prime}\right) h\left(f_{1}\right)=f_{2}^{\prime} f_{2} \text {. }
$$

Consider now the following diagram:

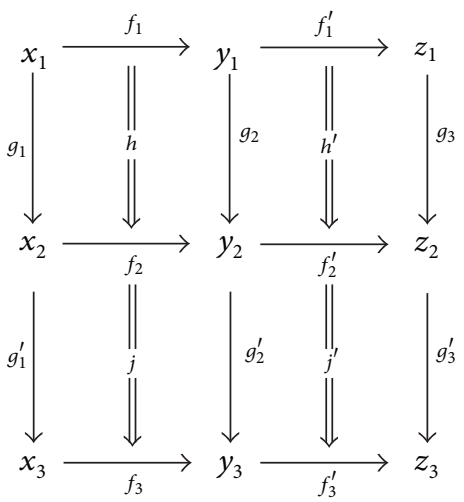

The morphisms of $\mathbb{F}(\mathbf{C})_{0}$ thus have two laws of composition: ${ }^{\circ} V$ and ${ }^{\circ} H$. As we see below, these compositions obey a consistency condition (28), which thereby specifies a double category $[10,11, \S \mathrm{I} .5]$.

Proposition 3. The morphisms of $\mathbb{F}(\mathbf{C})_{0}$ form a double category under the laws of composition ${ }^{\circ} \mathrm{V}$ and ${ }_{\mathrm{O}} \mathrm{H}$ in the sense that for diagram (27), with notation as explained above,

$$
\left(u_{j^{\prime} \circ H} u_{j}\right){ }^{\circ} V\left(u_{h^{\prime} \circ} u_{h}\right)=\left(u_{j^{\prime} \circ} u_{h^{\prime}}\right){ }^{\circ} H\left(u_{j}{ }^{\circ} V u_{h}\right) \text {, }
$$

for all morphisms $u_{j^{\prime}}, u_{j}, u_{h}, u_{h^{\prime}}$ in $\operatorname{Mor}\left(\mathbb{F}(\mathbf{C})_{0}\right)$ for which the compositions on both sides of (28) are meaningful.

Proof. Denote by $u_{h}$ the morphism of $\mathbb{F}(\mathbf{C})_{0}$ specified by the upper left square in (27), by $u_{h^{\prime}}$ the morphism specified by the upper right square, by $u_{j}$ the morphism specified by the lower left square, and, lastly, by $u_{j^{\prime}}$ the morphism specified by the lower right square.
Let $f \in \operatorname{Mor}\left(x_{1}, z_{1}\right)$. Then,

$$
\begin{aligned}
\left(\left(u_{j^{\prime} \circ} u_{j}\right){ }^{\circ} V\left(u_{h^{\prime} \circ} u_{h}\right)\right)(f)= & \left(u_{j^{\prime} \circ} u_{j}\right) \\
& \times\left(h^{\prime}\left(f f_{1}^{-1}\right) f_{2}\right) \\
= & j^{\prime}\left(h^{\prime}\left(f f_{1}^{-1}\right)\right) f_{3}, \\
\left(\left(u_{j^{\prime} \circ} u_{h^{\prime}}\right){ }^{\circ}{ }_{H}\left(u_{j} \circ{ } u_{h}\right)\right)(f)= & \left(\left(u_{j^{\prime} \circ V} u_{h^{\prime}}\right)\left(f f_{1}^{-1}\right)\right) f_{3} \\
= & j^{\prime}\left(h^{\prime}\left(f f_{1}^{-1}\right)\right) f_{3} .
\end{aligned}
$$

Comparing (29) and (30), we have the claimed equality (28).

Then, $\mathbb{F}(\mathbf{C})_{0}$ equipped with both laws of composition ${ }^{\circ} \mathrm{V}$ and ${ }^{\circ} H$ is a double category [11]. In the geometric context, this is expressed as a flatness condition for the connection $\omega_{\bar{A}, A, B}$ described in the Introduction; for more, see, for instance, [1, $3]$.

\section{Enrichment for Morphisms}

We continue with the notation and structures as before; $\mathbf{C}$ is a category and $\mathbb{F}(\mathbf{C})$ is the "fat" category described in Section 2. Now let $\mathbb{F}(\mathbf{C})_{1}$ be a subcategory of $\mathbb{F}(\mathbf{C})_{0}$, having the same objects but possibly fewer morphisms. The idea is that the hom-sets in $\mathbb{F}(\mathbf{C})$ could have additional structure; for example, if $\mathbf{C}$ has only one object $E_{p}$, a fiber of a vector bundle, then $\operatorname{Mor}\left(E_{p}, E_{p}\right)$ is a group under composition. The morphisms of $\mathbb{F}(\mathbf{C})_{1}$ could be required to be group automorphisms. We require that for any objects $x, y, z$ of $\mathbf{C}$ and isomorphism $g: y \rightarrow x$, the map

$$
r_{g}: \operatorname{Mor}(x, z) \longrightarrow \operatorname{Mor}(y, z): f \longmapsto f g
$$

is a morphism of $\mathbb{F}(\mathbf{C})_{1}$.

Proposition 4. Let $\mathbb{F}(\mathbf{C})_{1}$ be any subcategory of $\mathbb{F}(\mathbf{C})_{0}$ having the same objects as $\mathbb{F}(\mathbf{C})_{0}$, and satisfying the condition (31) as explained above. Both horizontal and vertical composites of morphisms in $\mathbb{F}(\mathbf{C})_{1}$ are in $\mathbb{F}(\mathbf{C})_{1}$. Thus, $\mathbb{F}(\mathbf{C})_{1}$ is a double category.

Proof. The consistency condition between horizontal and vertical compositions has already been checked in Proposition 3. Thus, we need only to check that horizontal composition, specified in (25) as

$$
h^{\prime \prime}: \operatorname{Mor}\left(x_{1}, z_{1}\right) \longrightarrow \operatorname{Mor}\left(x_{2}, z_{2}\right): f \longmapsto h^{\prime}\left(f f_{1}^{-1}\right) h\left(f_{1}\right) \text {, }
$$

is a morphism of $\mathbb{E}(\mathbf{C})_{1}$, for all invertible $f_{1} \in \operatorname{Mor}\left(x_{1}, y_{1}\right)$ and all $h: \operatorname{Mor}\left(x_{1}, y_{1}\right) \rightarrow \operatorname{Mor}\left(x_{1}^{\prime}, y_{1}^{\prime}\right), h^{\prime} \in \operatorname{Mor}\left(y_{1}, z_{1}\right) \rightarrow$ $\operatorname{Mor}\left(y_{1}^{\prime}, z_{1}^{\prime}\right)$ morphisms in $\mathbb{F}(\mathbf{C})_{1}$. Observe that

$$
h^{\prime \prime}(f)=h^{\prime}\left(f f_{1}^{-1}\right) h\left(f_{1}\right)=r_{h\left(f_{1}\right)} \circ h^{\prime} \circ r_{f_{1}^{-1}}(f) \text {, }
$$

where the notation $r_{g}$ is as in (31). Thus, $h^{\prime \prime}$ is a composite of morphisms in $\mathbb{F}(\mathbf{C})_{1}$. 


\section{Monoidal Structures}

In this section we will explore some algebraic structural enhancements of the fattened category $\mathbb{F}(\mathbf{C})_{0}$. The discussion is motivated by intrinsic algebraic considerations, but we discuss briefly now the relationship with the geometric context.

Consider the very special case where $\mathbf{C}$ is the category with only one object $E_{o}$, the fiber over a fixed point $o$ in a vector bundle, and a morphism $f: E_{o} \rightarrow E_{o}$ is a an ordered pair as follow:

$$
f=(c, T),
$$

consisting of a piecewise smooth loop $c$ based at $o$ (with backtracks erased) along with a linear map $T: E_{o} \rightarrow E_{o}$ representing parallel transport around the loop. For $\mathbb{F}(\mathbf{C})_{0}$ in this special case, a morphism $h: \operatorname{Mor}\left(E_{o}, E_{o}\right) \rightarrow$ $\operatorname{Mor}\left(E_{o}, E_{o}\right)$ arises from paths of paths along with a linear map $\operatorname{End}\left(E_{o}\right) \rightarrow \operatorname{End}\left(E_{o}\right)$, where $\operatorname{End}\left(E_{o}\right)$ is the vector space of all linear maps $E_{o} \rightarrow E_{o}$. Each hom-set $\operatorname{Mor}\left(E_{o}, E_{o}\right)$ is a monoid: composition

$$
\begin{aligned}
\operatorname{Mor}\left(E_{o}, E_{o}\right) \times \operatorname{Mor}\left(E_{o}, E_{o}\right) & \longrightarrow \operatorname{Mor}\left(E_{o}, E_{o}\right):\left(f, f^{\prime}\right) \\
& \longmapsto f \otimes f^{\prime}
\end{aligned}
$$

is given by concatenation of loops along with ordinary composition of linear maps in $\operatorname{End}\left(E_{o}\right)$ :

$$
(c, T) \otimes\left(c^{\prime}, T^{\prime}\right)=\left(c * c^{\prime}, T \circ T^{\prime}\right),
$$

where $c * c^{\prime}$ is the loop $c^{\prime}$ followed by the loop $c$. (Since this discussion is primarily for motivation, we leave out technical details of "backtrack erasure.")

Turning to the abstract setting, we assume henceforth that $\mathrm{C}$ is a monoidal category. This means that there is a bifunctor

$$
\otimes: \mathbf{C} \times \mathbf{C} \longrightarrow \mathbf{C}
$$

and there is an identity object 1 in $\mathbf{C}$ for which certain natural coherence conditions hold as we now describe. In addition, there exists a natural isomorphism $\alpha$, the associator, which associates to any of the objects $A, B, C$ of $\mathbf{C}$ an isomorphism

$$
\alpha_{A, B, C}:(A \otimes B) \otimes C \longrightarrow A \otimes(B \otimes C)
$$

such that the following diagram commutes:

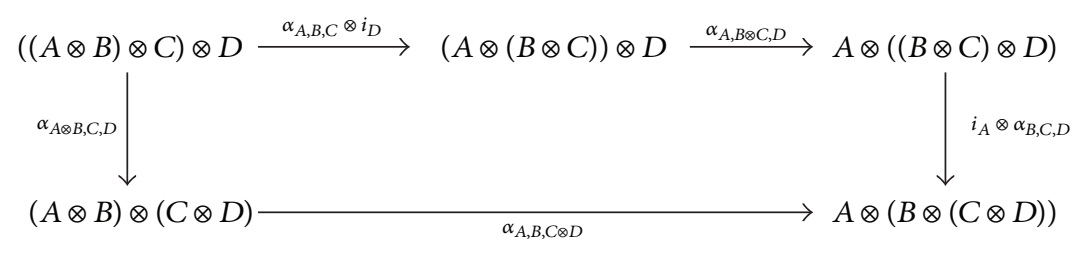

There are also natural isomorphisms $l$ and $r$, the left and right unitors, associating to each object $A$ in $\mathbf{C}$ morphisms

$$
l_{A}: 1 \otimes A \longrightarrow A, \quad r_{A}: A \otimes 1 \longrightarrow A
$$

such that

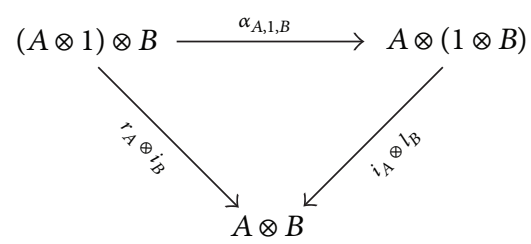

commutes for all objects $A$ and $B$ in $\mathbf{C}$.

Note that naturality means there are certain other conditions as well. For example, that the left unitor is a natural transformation means that for any morphism $x \stackrel{f}{\rightarrow} y$ in $\mathbf{C}$ the diagram

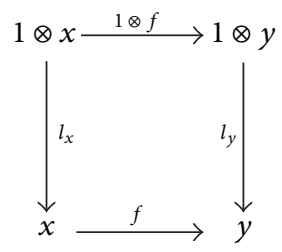

commutes; here, in the upper horizontal arrow, 1 is the unique morphism $i_{1}: 1 \rightarrow 1$ in $\mathbf{C}$.

We now define a product on the objects of $\mathbb{F}(\mathbf{C})$

$\operatorname{Obj}(\mathbb{F}(\mathbf{C})) \times \operatorname{Obj}(\mathbb{F}(\mathbf{C})) \longrightarrow \operatorname{Obj}(\mathbb{F}(\mathbf{C})):(u, v) \longmapsto u \otimes v$ 
as follows:

$$
\left(x_{1} \stackrel{f_{1}}{\longrightarrow} y_{1}\right) \otimes\left(x_{2} \stackrel{f_{2}}{\longrightarrow} y_{2}\right) \stackrel{\text { def }}{=} x_{1} \otimes x_{2} \stackrel{f_{1} \otimes f_{2}}{\longrightarrow} y_{1} \otimes y_{2} .
$$

In the fat category $\mathbb{F}(\mathbf{C})$, we then have associators and unitors as follows. First, the unit is

$$
1_{\mathbb{F}}=1 \stackrel{i_{1}}{\longrightarrow} 1,
$$

where 1 denotes the identity object in $\mathbf{C}$ and $i_{1}$ the identity map on 1 . We will often denote $i_{1}$ also simply as 1 , the meaning being clear from context. For any object $x \stackrel{f}{\rightarrow} y$, there is the left unitor

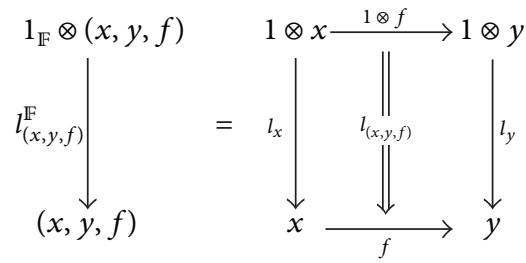

where the mapping

$$
l_{(x, y, f)}: \operatorname{Mor}(1 \otimes x, 1 \otimes y) \longrightarrow \operatorname{Mor}(x, y): \phi \longmapsto l_{y} \phi l_{x}^{-1}
$$

takes $1 \otimes f$ to $f$, as follows from the remarks made above for (42). The right unitor is

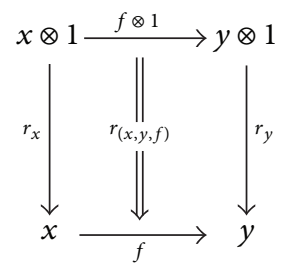

where

$$
r_{(x, y, f)}: \operatorname{Mor}(x \otimes 1, y \otimes 1) \longrightarrow \operatorname{Mor}(x, y): \phi \longmapsto r_{y} \phi r_{x}^{-1} .
$$

Again, this is indeed a morphism in $\mathbb{F}(\mathbf{C})$ by essentially the same argument that was used above in (46) for the left unitor.

The associator in $\mathbb{F}(\mathbf{C})$ is given as follows. Consider objects $x_{i} \stackrel{f_{i}}{\longrightarrow} y_{i}$ in $\mathbb{F}(\mathbf{C})$, for $i \in\{1,2,3\}$. The fact that $\alpha$ is a natural transformation means that the diagram

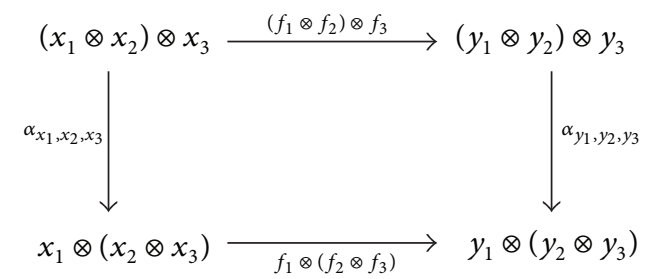

is commutative. Hence, by the first half of Lemma 2, this induces a morphism
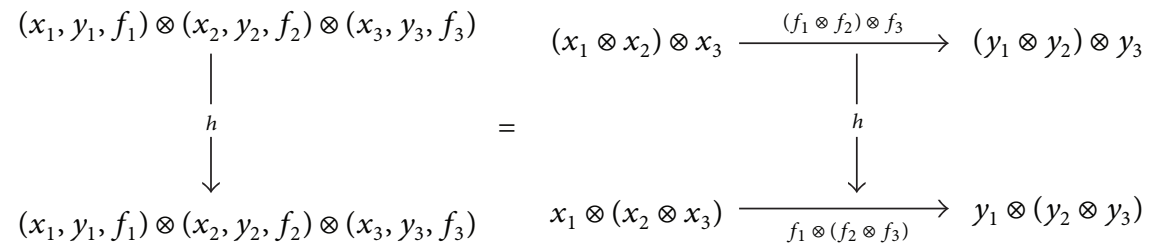

in $\mathbb{F}(\mathbf{C})$. In fact, $h$ is an isomorphism since the vertical arrows in (50) are isomorphisms.

We prove the coherence condition for unitors. For this we have the following diagram:

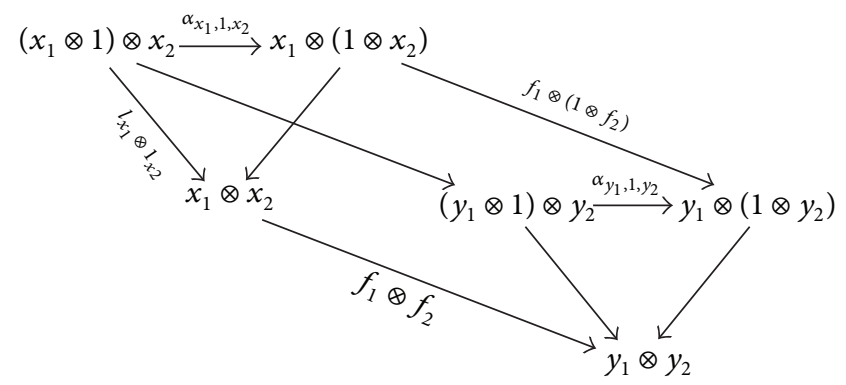

The two triangles at the two ends of this "trough" commute because of coherence in $\mathrm{C}$, the top rectangle also commutes because of the naturality of $\alpha$. Then, it is entertaining to check that the two rectangular "slanted sides" are also commutative. In fact, the slant side on the left is

$$
\begin{aligned}
r_{\left(x_{1}, y_{1}, f_{1}\right)}^{\mathbb{F}} \otimes 1_{\left(x_{2}, y_{2}, f_{2}\right)} & :\left(\left(x_{1}, y_{1}, f_{1}\right) \otimes 1^{\mathbb{F}}\right) \otimes\left(x_{2}, y_{2}, f_{2}\right) \\
& \longrightarrow\left(x_{1}, y_{1}, f_{1}\right) \otimes\left(x_{2}, y_{2}, f_{2}\right)
\end{aligned}
$$

as a morphism in $\mathbb{F}(\mathbf{C})$, and the slant side on the right is

$$
1_{\left(x_{1}, y_{1}, f_{1}\right)} \otimes l_{\left(x_{2}, y_{2}, f_{2}\right)}^{\mathbb{F}} .
$$


Thus, viewed as a diagram in $\mathbb{F}(\mathbf{C})$, the "trough" looks like

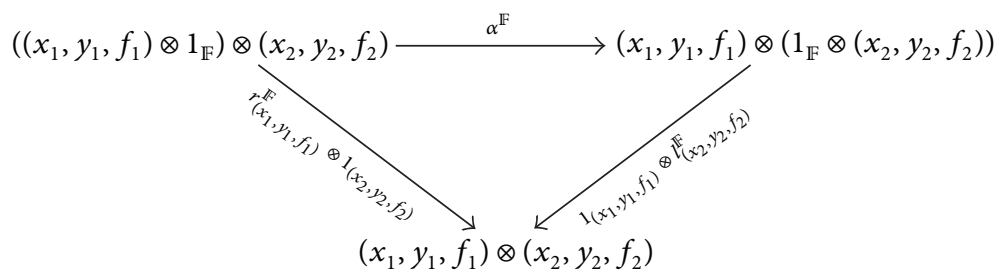

Since the trough commutes in C, so does its avatar (55) in $\mathbb{F}(\mathrm{C})$, thanks to the second half of Lemma 2 . This verifies the coherence property in $\mathbb{F}(\mathbf{C})$ involving the unitors.

Now, we turn to coherence for the associators. In the following diagram, where we leave out the $\otimes$ products for ease of viewing, the slant arrows are all tensor products of the $f_{i}$ and the horizontal and vertical arrows are various associators:

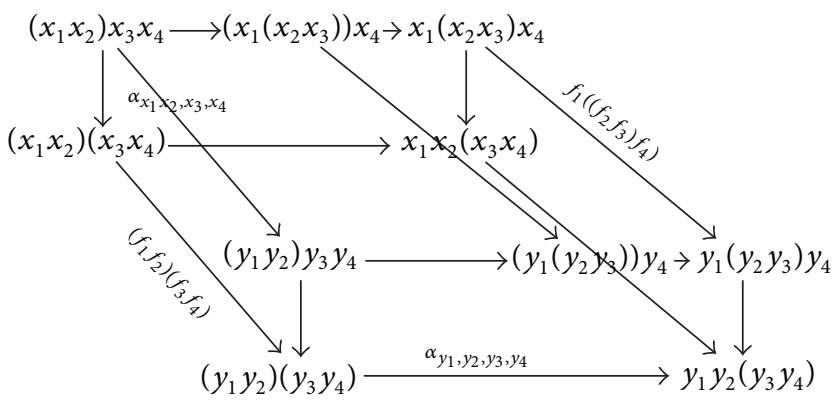

Coherence in the monoidal category $\mathbf{C}$ implies that the two rectangles at the end of this box are commutative, as mentioned earlier. Naturality of the associator implies that the top, bottom, and sides are also commutative. Thus, the entire diagram is commutative. If we abbreviate the objects in $\mathbb{F}(\mathbf{C})$ as

$$
X_{i}=\left(x_{i}, y_{i}, f_{i}\right),
$$

for $i \in\{1,2,3,4\}$, we can read the full diagram as a diagram in the category $\mathbb{F}(\mathbf{C})$ as follows:

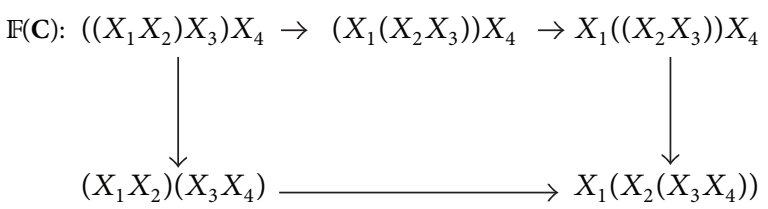

As a diagram in $\mathbb{F}(\mathbf{C})$, this is commutative, by Lemma 2. This establishes coherence of the associator in $\mathbb{F}(\mathbf{C})$.

We have completed the proof of Proposition 5.

Proposition 5. Suppose that $\mathbf{C}$ is a monoidal category and let $\mathbb{F}(\mathbf{C})$ be the category specified above in the context of (11). Then, with tensor product as defined in (44), $\mathbb{F}(\mathbf{C})$ satisfies all conditions of a monoidal category at the level of objects.

\section{Concluding Remarks}

In this paper, we have presented certain "fattened" categories $\mathbb{F}(\mathbf{C}), \mathbb{F}(\mathbf{C})_{0}$, and $\mathbb{F}(\mathbf{C})_{1}$ constructed out of a given category $\mathbf{C}$; the morphisms of $\mathbb{F}(\mathbf{C})_{0}$ form a double category. It is shown how a monoidal structure on $\mathbf{C}$ induces a multiplication on the objects of $\mathbb{F}(\mathbf{C})$ that satisfies certain coherence properties.

\section{Acknowledgments}

A. Lahiri acknowledges research support from Department of Science and Technology, India, under Project no. SR/S2/HEP0006/2008. A. N. Sengupta acknowledges research support at an earlier stage of this work from US NSF Grant DMS0601141. The authors have used macros developed by Paul Taylor to draw all diagrams.

\section{References}

[1] S. Chatterjee, A. Lahiri, and A. N. Sengupta, "Parallel transport over path spaces," Reviews in Mathematical Physics, vol. 22, no. 9, pp. 1033-1059, 2010.

[2] T. Lévy, "Two-Dimensional Markovian Holonomy Fields," Astérisque, vol. 329, 2010.

[3] J. Baez, "Higher Yang-Mills Theory," unpublished, http://arxiv .org/abs/hep-th/0206130. 
[4] J. Baez and U. Schreiber, "Higher gauge theory, in categories in Algebra, geometry and mathematical physics," in Proceedings of the Contemporary Mathematics (AMS '07), A. Davydov, Ed., American Mathematical Society, 2007.

[5] J. Baez and U. Schreiber, "Higher Gauge Theory II: 2-connections on 2-bundles," unpublished, http://arxiv.org/abs/hepth/0412325.

[6] F. Girelli and H. Pfeiffer, "Higher gauge theory-differential versus integral formulation," Journal of Mathematical Physics, vol. 45, no. 10, pp. 3949-3971, 2004.

[7] A. Lahiri, "Surface holonomy and gauge 2-group," International Journal of Geometric Methods in Modern Physics, vol. 1, no. 4, pp. 299-309, 2004.

[8] J. Baez, "Quantum quandaries: a category-theoretic perspective," in The Structural Foundations of Quantum Gravity, pp. 240-265, Oxford University Press, Oxford, UK, 2006.

[9] M. Shulman, "Constructing symmetric monoidal bicategories," unpublished, http://arxiv.org/abs/1004.0993.

[10] S. Mac Lane, Categories for the Working Mathematician, Springer, 1971.

[11] G. M. Kelly and R. Street, "Reviews of the elements of 2categories," in Category Seminar, vol. 420 of Lecture Notes in Mathematics, pp. 75-103, Springer, Berlin, Germany, 1974. 

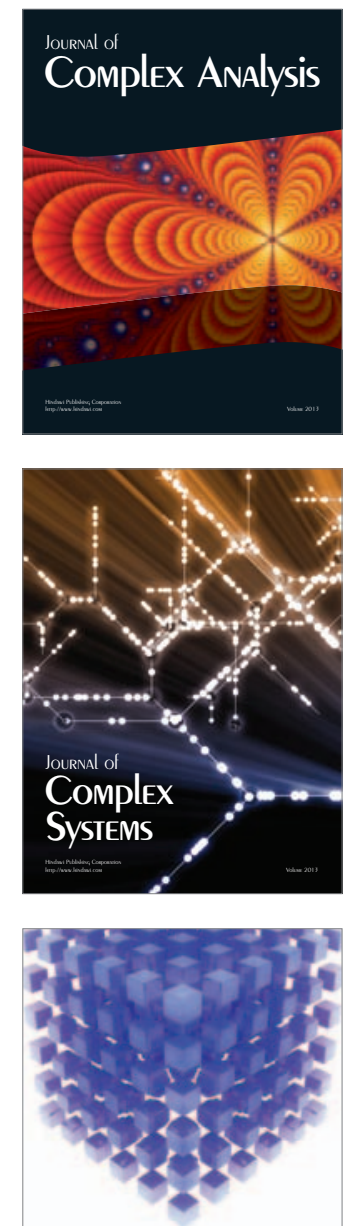

Mathematical Problems in ENGiNEERING
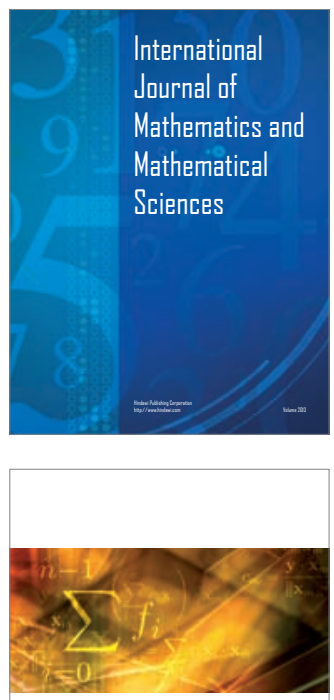

ISRN

Applied

Mathematics
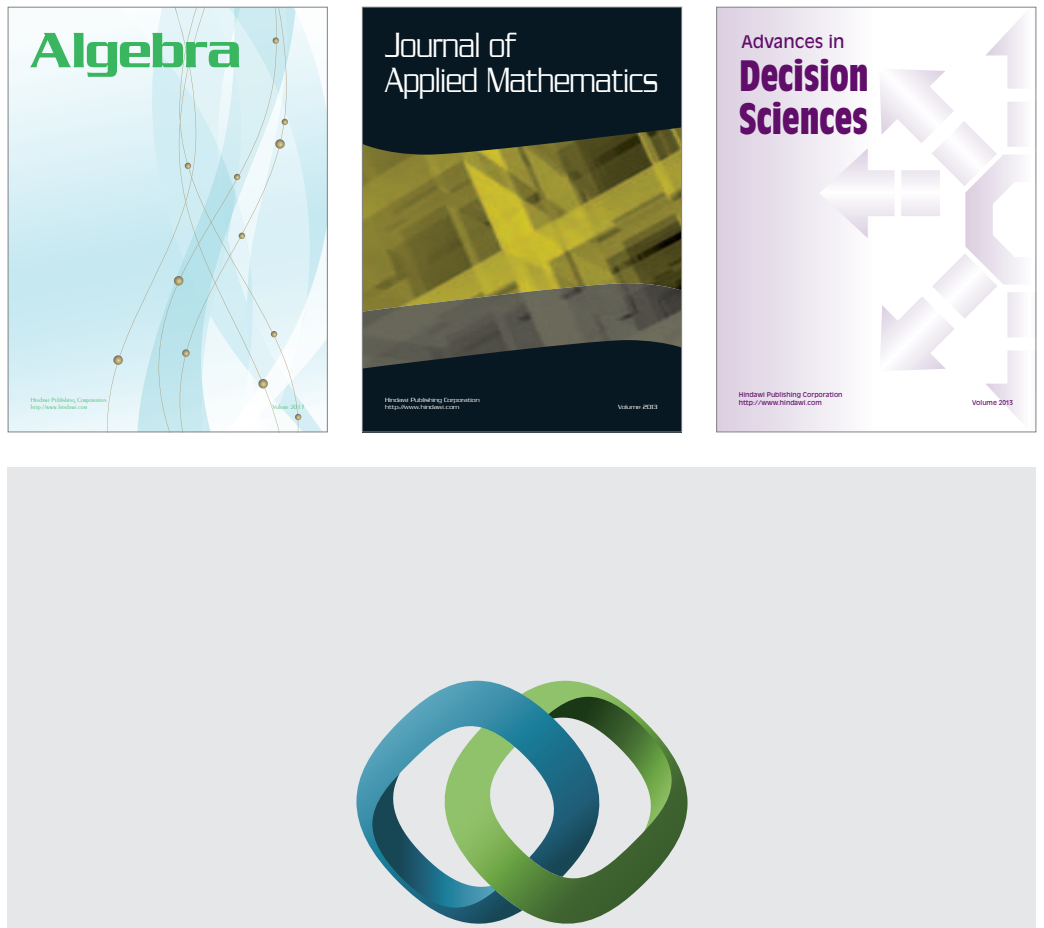

\section{Hindawi}

Submit your manuscripts at http://www.hindawi.com

Game Theory
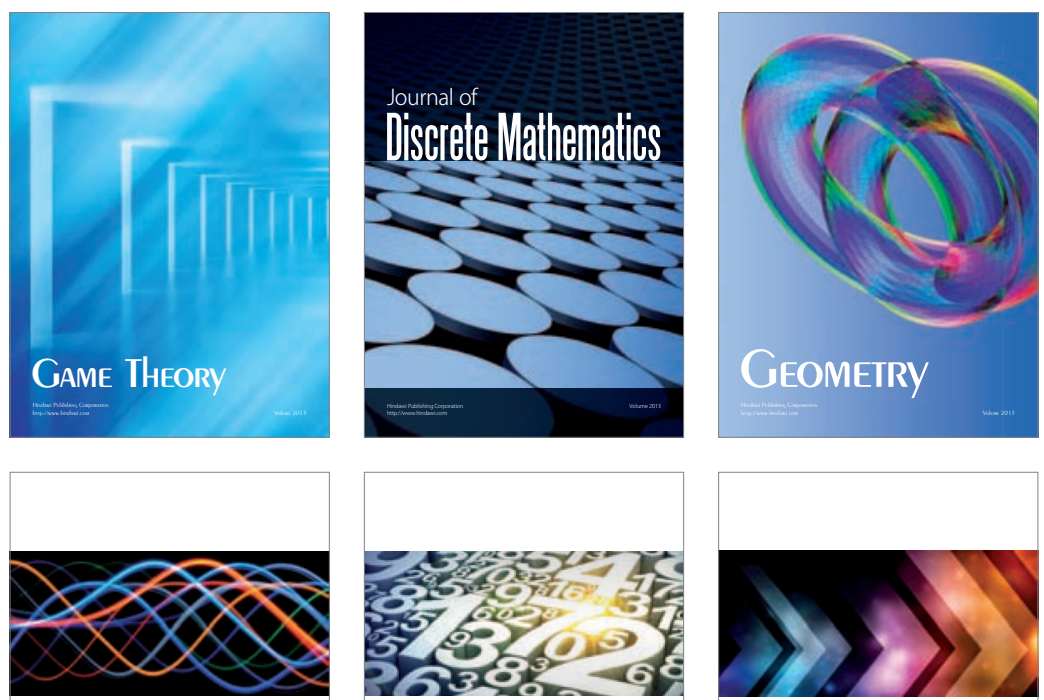

ISRN

Mathematical

Physics

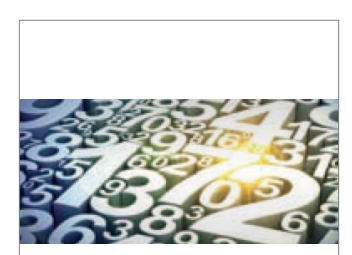

ISRN

Combinatorics

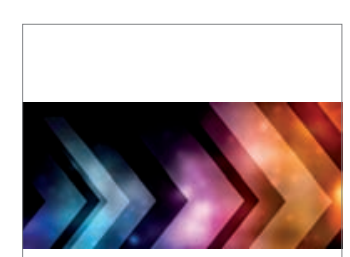

ISRN

Operations

Research
ISRN

Computational

Mathematics

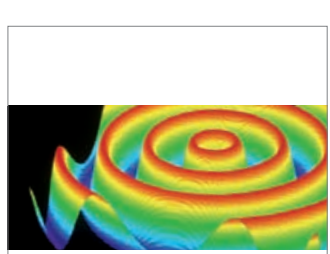

Abstract and

Applied Analysis
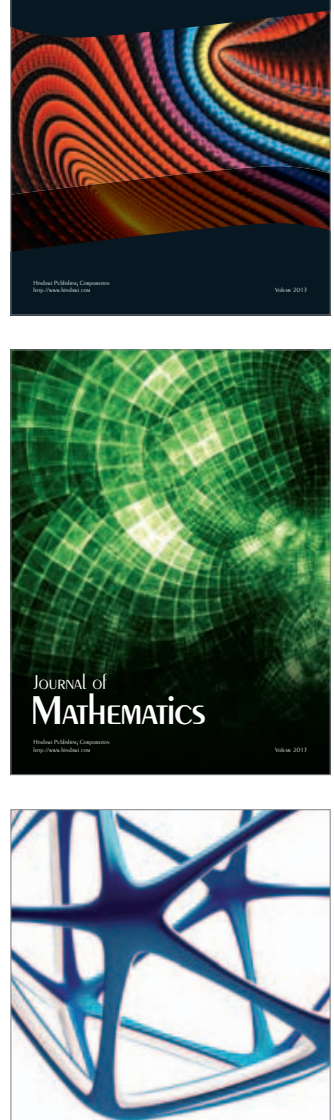

Ilownul of
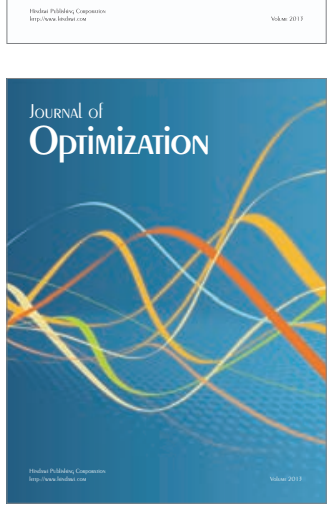

Mathematics 


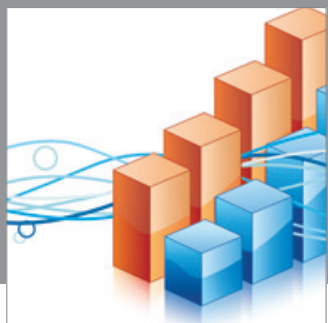

Advances in

Operations Research

mansans

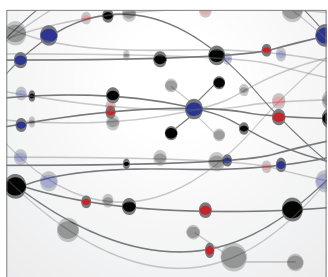

The Scientific World Journal
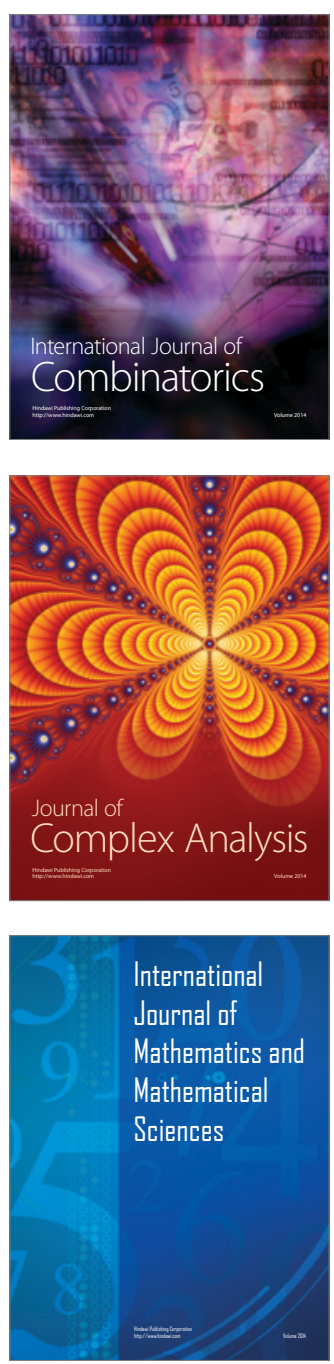
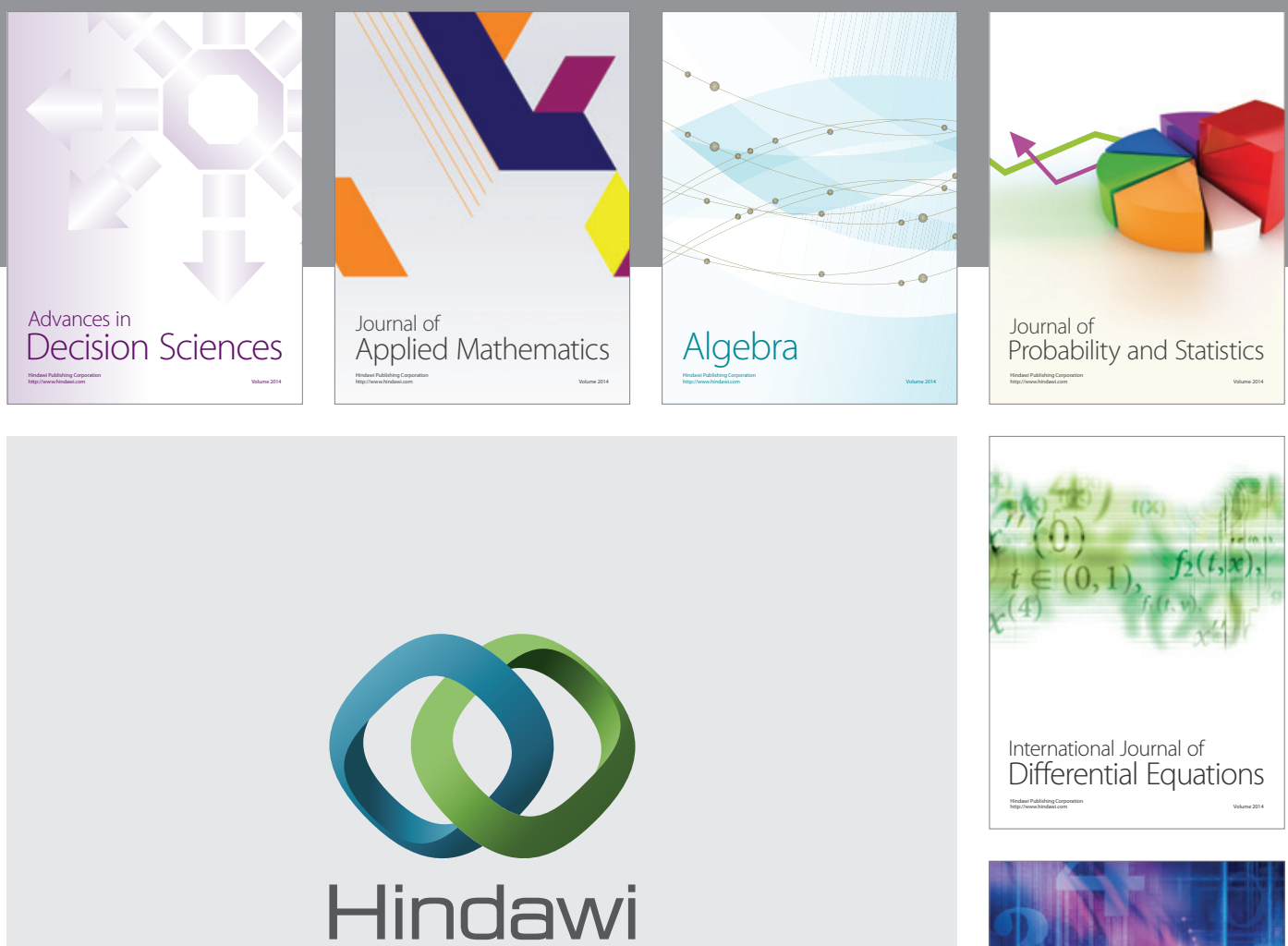

Submit your manuscripts at http://www.hindawi.com
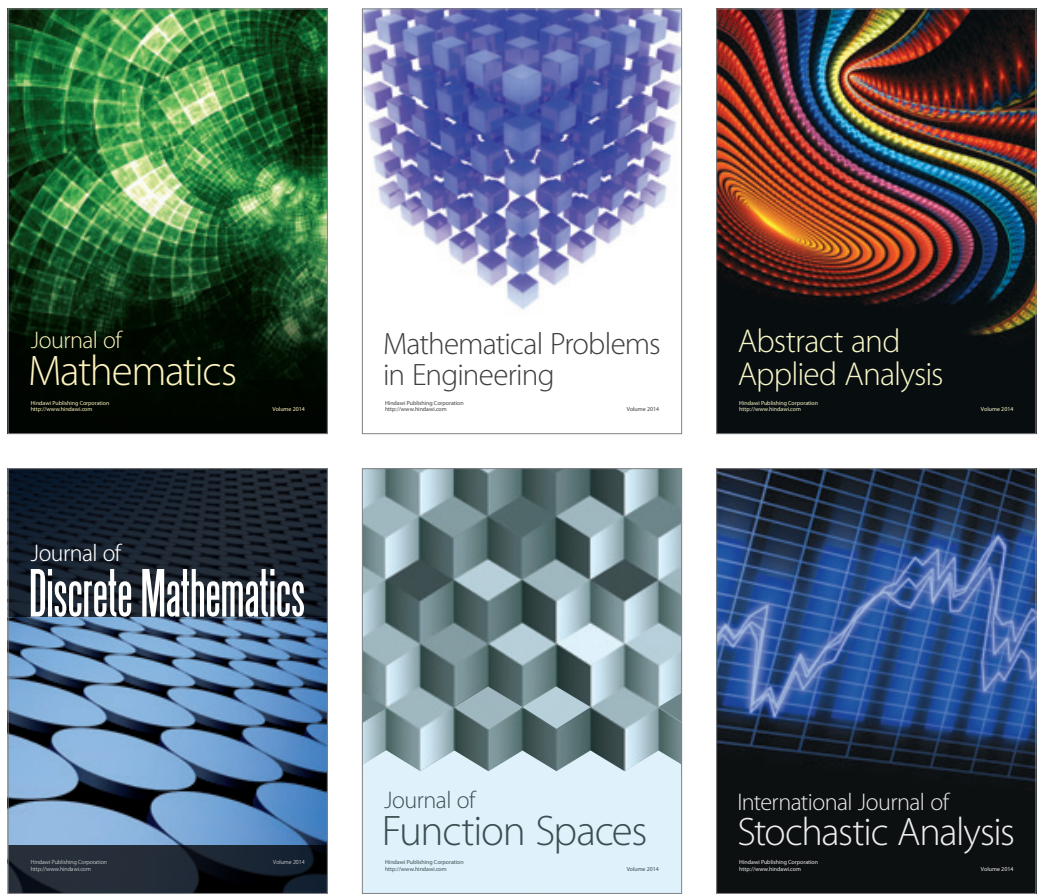

Journal of

Function Spaces

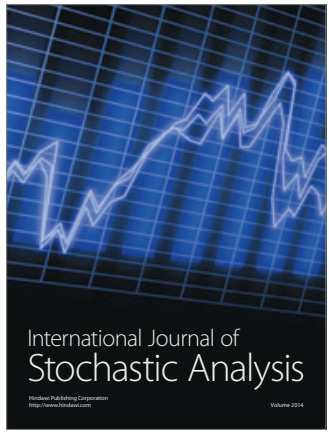

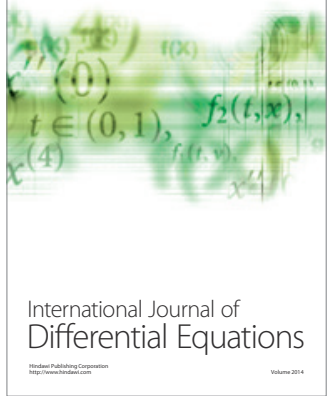
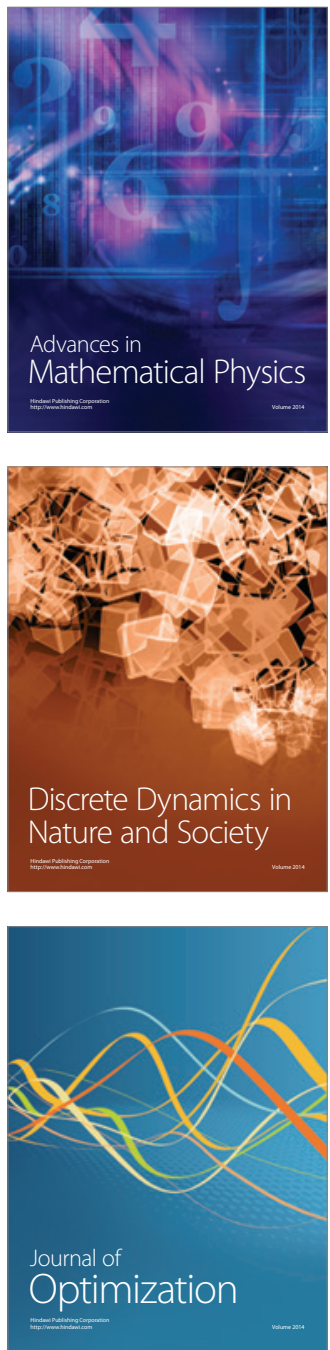